\title{
Effects of the COVID-19 Pandemic on Cardiac Surgery Practice and Outcomes
}

\author{
Kirun Gopal, M.Ch., Neethu Krishna, M.H.A., Rajesh Jose, M.Ch., Surya Sree Chitra Biju, B.Sc., \\ Jaya Suriya Pichandi, B.Sc., Praveen Kerala Varma, M.Ch.
}

Department of Cardiovascular and Thoracic Surgery, Amrita Institute of Medical Sciences and Research Center, Amrita Vishwa Vidyapeetham (Amrita University), Kochi, India

\section{ARTICLE INFO}

Received October 6, 2021

Revised November 22, 2021

Accepted December 13, 2021

Corresponding author

Kirun Gopal

Tel $91-4846681559$

Fax 91-9495803314

E-mail kirungopal@aims.amrita.edu

ORCID

https://orcid.org/0000-0002-9622-4230
Background: While the coronavirus disease 2019 (COVID-19) pandemic has affected all aspects of health care, its impact on cardiac surgical practice and outcomes is yet to be determined. We compared the outcomes of our cardiac surgical practice from the past year during the pandemic to those in a similar pre-pandemic period.

Methods: Retrospective data were collected from 307 patients who were involved in all adult cardiac surgical procedures performed between March 2020 and February 2021, which was considered the pandemic period, at Amrita Institute of Medical Sciences, India. These were compared with data from the 1-year period between March 2019 and February 2020. During that earlier period, 491 patients underwent surgery, and the surgical outcomes were assessed. Outpatient visit data were also collected to evaluate the effect of COVID-19 on outpatient follow-up visits.

Results: A 37\% decrease in surgical case volume was observed during the study period. No difference was found in operative mortality between the 2 time periods ( $3.3 \%$ vs. $2.6 \%$, $\mathrm{p}=0.383)$. Overall postoperative complications were less frequent during this period, at $23 \%$ compared to $38 \%$ the previous year $(p<0.001)$.

Conclusion: The COVID-19 pandemic caused a dramatic decrease in surgical volume and outpatient medical follow-up. However, the pandemic and its attendant social restrictions did not yield a significant change in the surgical outcomes of our patients. Hence, it is reasonable to continue cardiac surgical care during global health crises, and this can be done with good results.

Keywords: COVID-19, Pandemic, Cardiac surgery, Outcomes

\section{Introduction}

The first case of coronavirus disease 2019 (COVID-19) caused by the novel severe acute respiratory syndrome coronavirus 2 (SARS-CoV-2) was reported in December 2019 in Wuhan, China. Since then, the disease has spread globally and was declared a pandemic on March 11, 2020 by the World Health Organization. The first patient with COVID-19 in India was diagnosed in January 2020. An unprecedented national lockdown was instituted on March 25,2020 , resulting in the curtailing of all regular activities. During the second half of the year, the restrictions were eased in a phased manner commensurate with the declining trend of infections. Despite these measures, by April
2021, India had the second largest burden of COVID-19 cases in the world.

This once-in-a-century event has left its mark on every aspect of life, including cardiovascular care. Numerous reports have demonstrated increased cardiovascular mortality during the pandemic $[1,2]$. This has been attributed to a delay in regular medical care due to restrictions, the overburdening of medical infrastructure, and fear among patients to seek medical care during the pandemic $[3,4]$. Consequently, some experts were concerned about the possibility of an increased frequency of complications related to delayed care. Potential impacts include patients with a greater degree of ventricular dysfunction due to inadequate or delayed treatment for myocardial infarction, a 
greater number of mechanical complications of myocardial infarction, and patients in more advanced stages of heart failure [5-7]. Hence, it is only natural to assume that cardiac surgical outcomes may similarly be adversely affected, as patients may present at more advanced stages of disease. Other groups have also raised this concern $[8,9]$. The fear of contracting COVID-19 may also make patients reluctant to attend their regular outpatient follow-up appointments. Very few, and sporadic, early reports of the effects of the pandemic on cardiac surgery outcomes have been published over the past year.

The aim of the present study was to evaluate the effects of the COVID-19 pandemic and the associated social restrictions on adult cardiac surgical care and outcomes at a tertiary referral center in comparison to the pre-pandemic period.

\section{Methods}

\section{Study design}

In this retrospective single-center observational study, all adult cardiac surgery patients operated on or treated in the outpatient department between March 2020 and February 2021 (designated as the pandemic period) were compared to those in a similar pre-pandemic time period between March 2019 and February 2020. All data were collected from our departmental database, which includes all pertinent preoperative, postoperative, and mortality-related data of all operated patients. Any missing values were obtained prospectively from patient records. Outpatient follow-up data were collected from the electronic medical records of the hospital.

\section{Aim of the study}

The primary aim of the study was to evaluate the effects of the COVID-19 pandemic and the associated social restrictions on adult cardiac surgical care and outcomes at a tertiary referral center in comparison to a pre-pandemic historical control period.

A secondary aim was to identify whether an increase occurred in the incidence of left ventricle (LV) dysfunction among patients who underwent isolated coronary artery bypass surgery (CABG) during the pandemic period. In this report, we also present our experiences of managing patients with COVID-19 who underwent cardiac surgery during this period. The Institutional Review Board at Amrita Institute of Medical Sciences approved the study
(ECASM-AIMS-2021-223 dated 18-05-2021), and the requirement for individual patient consent was waived.

\section{Pandemic context and hospital policy regarding COVID-19}

Our center is in the state of Kerala in South India, which is currently the second most heavily-affected state with regard to the number of COVID-19 cases. The hospital is a not-for-profit 1,350-bed tertiary referral center. Very early in the pandemic, a separate wing was designated as the COVID-19 wing and included a designated intensive care unit (ICU) and wards.

\section{Inpatient management}

At the onset of the pandemic, members of the hospital administration met with the heads of the surgical departments to establish a common surgical pathway. Patients were categorized based on their risk of COVID-19 infection. Procedures were also categorized as elective, semielective, or emergency. Based on this, patients were given a period of preoperative quarantine in the hospital guest house based on surgical urgency and 2 negative reversetranscription polymerase chain reaction (RT-PCR) tests before admission to the hospital. Patients requiring emergency surgery were admitted to a designated common ICU or ward for possible COVID-19 cases after submission of the RT-PCR test. If the test was negative, the patient was moved to the regular ICU or directly to the operating theater if required. Patients whose cases were too severe to wait for the RT-PCR result, such as those with acute aortic dissection, were moved directly to 1 of 2 designated negative-pressure COVID-19 theaters, which had been converted in preparation for such emergencies. Procedures were also classified by risk of transmission into 3 tiers, based on the possibility of false-negative RT-PCR or the patient being in the window period; the level of personal protective equipment was chosen accordingly. COVID-19-positive patients were moved through a dedicated corridor from the COVID-19 ICU to the theater and back to the COVID19 ICU after surgery. This led to COVID-19-positive patients from multiple departments being treated postoperatively in a common ICU, which raised some logistical issues. Overall, inpatient COVID-19-related treatment was handled by the infectious disease department, with ICU support provided by the critical care team. 


\section{Cardiac surgery department}

As part of the hospital protocol, we formulated our departmental procedures for triaging patients early in the pandemic in accordance with national and international society guidelines $[10,11]$. The hospital department includes 3 divisions: adult cardiac, pediatric cardiac, and thoracic-vascular. In the adult cardiac division, we generally operate on between 500-600 patients per year, and approximately $1,000-1,200$ operations are performed per year in the department. We cater to the entire breadth of adult cardiac surgery and accept all patients with indications for surgery after explaining the risks. Additionally, we are one of only a few centers in the state that treat aortic dissections, active endocarditis, and patients with extensive comorbidities.

\section{Statistical analysis}

Data were analyzed using IBM SPSS ver. 20.0 software (IBM Corp., Armonk, NY, USA). The results are provided as mean \pm standard deviation for continuous variables and as frequency and percentage for categorical variables. To assess the statistical significance of the difference in the proportion of categorical variables between the years 20192020 and 2020-2021, the chi-square test was used. To evaluate the statistical significance of the difference in mean age between those years, the Student t-test was utilized. Finally, to test the statistical significance of the differences in the mean and median of European System for Cardiac Operative Risk Evaluation (EuroSCORE) II, preoperative creatinine, and postoperative creatinine values between the relevant years, the Mann-Whitney $U$ test was used. A p-value of $<0.05$ was considered to indicate statistical significance.

\section{Results}

During the 1-year study period, 307 patients underwent cardiac surgery in the adult cardiac surgery division. This represents a $37 \%$ drop in average surgical volume compared to the previous year (Fig. 1). During a similar 1-year period prior to the pandemic, 491 patients underwent cardiac surgery. In the pandemic period, the hospital had 1,166 COVID-19 admissions, and 88 people died from the disease. The mean age of the patients was $59 \pm 11.4$ years, and $75 \%$ were male. The demographic profile was similar between the 2 years.

Other preoperative characteristics compared between

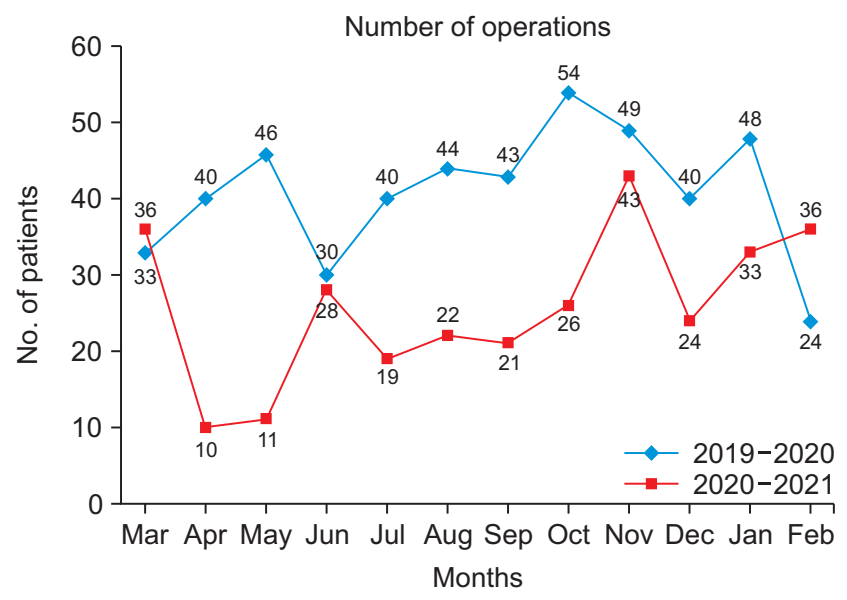

Fig. 1. Surgical volume in the pandemic (2020-2021) and prepandemic (2019-2020) periods.

the 2 time periods showed no significant differences, including EuroSCORE II $(\mathrm{p}=0.469)$, preoperative creatinine $(\mathrm{p}=0.587)$, and urgency of surgery $(\mathrm{p}=0.402)$. Table 1 shows the preoperative characteristics of the patients. The procedures performed during the study period are shown in Table 2. A subgroup analysis of patients undergoing isolated CABG was performed to identify whether a difference was present in the incidence of LV dysfunction in patients presenting for CABG. This showed no significant difference $(\mathrm{p}=0.801)$ (Table 3$)$.

Postoperative complications were observed in $23 \%$ of patients during the study period. This was significantly lower than the complication rate of the pre-pandemic time period, which was $38 \%(\mathrm{p}<0.001)$. The incidence of surgical site infection was also lower during the study period, at $1 \%$ compared to $4.9 \%$ in the previous year $(\mathrm{p}=0.002)$. Other postoperative complications as listed in Table 4 did not significantly differ between the time periods. Operative mortality was similar during the 2 periods, at $3.3 \%$ during the pandemic period compared to $2.6 \%$ the previous year $(\mathrm{p}=0.616)$.

Outpatient visits were also 39\% lower during the pandemic period than during the prior year (Fig. 2).

We operated on 1 patient with a known COVID-19 infection during this period, and 7 patients developed COVID-19 infection after surgery, 3 during the immediate postoperative period. All personnel involved in operating on the COVID-19-positive patient wore full personal protective equipment during the procedure. The patient with a known COVID-19 infection during surgery, who had a type A aortic dissection, died on postoperative day 10 , and 1 of the 3 patients who developed COVID-19 in the imme- 
Table 1. Baseline patient characteristics

\begin{tabular}{|c|c|c|c|}
\hline Characteristic & $2019-2020(n=491)$ & $2020-2021(n=307)$ & $p$-value \\
\hline Age $(y r)$ & $59.42 \pm 11.06$ & $58.34 \pm 11.91$ & 0.193 \\
\hline Sex & & & 0.709 \\
\hline Male & $372(75.8)$ & $229(74.6)$ & \\
\hline Female & $119(24.2)$ & $78(25.4)$ & \\
\hline Serum creatinine $(\mathrm{mg} / \mathrm{dL})$ & & & 0.587 \\
\hline Mean \pm SD & $1.09 \pm 0.54$ & $1.09 \pm 0.62$ & \\
\hline Median (IQR) & $1.01(0.84-1.19)$ & $0.99(0.84-1.17)$ & \\
\hline Left ventricular ejection fraction (\%) & & & 0.801 \\
\hline$\geq 50$ & $351(71.5)$ & $222(72.3)$ & \\
\hline$<50$ & $140(28.5)$ & $85(27.7)$ & \\
\hline EuroSCORE II & & & 0.469 \\
\hline Mean \pm SD & $2.13 \pm 2.59$ & $1.97 \pm 2.21$ & \\
\hline Median (IQR) & $1.30(0.90-2.23)$ & $1.30(0.82-2.19)$ & \\
\hline Urgency of surgery & & & 0.402 \\
\hline Elective & $450(91.6)$ & $276(89.9)$ & \\
\hline Emergency & $41(8.4)$ & $31(10.1)$ & \\
\hline
\end{tabular}

Values are presented as mean \pm standard deviation, number of patients (\%), or median (IQR), unless otherwise stated. A p-value $<0.05$ indicates statistical significance.

SD, standard deviation; IQR, interquartile range; EuroSCORE, European System for Cardiac Operative Risk Evaluation.

Table 2. Procedures performed

\begin{tabular}{lcc}
\hline \multicolumn{1}{c}{ Procedure } & 2019-2020 & 2020-2021 \\
\hline Coronary artery bypass graft & 325 & 191 \\
Aortic valve replacement & 47 & 28 \\
Mitral valve replacement & 32 & 18 \\
Double valve replacement & 8 & 9 \\
Mitral valve repair & 34 & 20 \\
Aortic surgery & 17 & 20 \\
Others & 28 & 21 \\
\hline
\end{tabular}

Table 3. LVEF in coronary artery bypass surgery patients

\begin{tabular}{cccc}
\hline Variable & $\begin{array}{c}2019-2020 \\
(\mathrm{n}=325)\end{array}$ & $\begin{array}{c}2020-2021 \\
(\mathrm{n}=191)\end{array}$ & p-value \\
\hline LVEF $(\%)$ & & & 0.478 \\
$\geq 50$ & $213(65.5)$ & $131(68.6)$ & \\
$<50$ & $112(34.5)$ & $60(31.4)$ & \\
\hline
\end{tabular}

Values are presented as number of patients (\%).

LVEF, left ventricular ejection fraction.

diate postoperative period who underwent a CABG died on day 9. Both patients succumbed due to COVID-19, with its associated inflammatory storm and progressive multiorgan failure. The other 2 patients recovered without requiring any further escalation in care. Four patients contracted COVID-19 infection after discharge but had uneventful recovery periods and were doing well at review.

Our surgical experience with patients with COVID-19 resembled the global data, with 2 of 4 patients succumbing
Table 4. Postoperative outcomes

\begin{tabular}{lccc}
\hline \multicolumn{1}{c}{ Variable } & $\begin{array}{c}2019-2020 \\
(\mathrm{n}=491)\end{array}$ & $\begin{array}{c}2020-2021 \\
(\mathrm{n}=307)\end{array}$ & p-value \\
\hline Complication & $186(37.9)$ & $71(23.1)$ & $<0.001^{*}$ \\
Re-exploration & $22(4.5)$ & $15(4.9)$ & 0.791 \\
Acute kidney injury & $87(17.7)$ & $47(15.3)$ & 0.376 \\
New-onset dialysis & $11(2.2)$ & $6(2.0)$ & 0.786 \\
Prolonged ventilation & $54(11.0)$ & $32(10.4)$ & 0.799 \\
Stroke & $6(1.2)$ & $3(1.0)$ & 1.000 \\
Reintubation & $29(5.9)$ & $16(5.2)$ & 0.679 \\
Mediastinitis & $7(1.4)$ & $1(0.3)$ & 0.249 \\
Surgical site infection & $24(4.9)$ & $3(1.0)$ & $0.006^{*}$ \\
Mortality & $13(2.6)$ & $10(3.3)$ & 0.616 \\
\hline
\end{tabular}

Values are presented as number of patients (\%).

${ }^{*} p<0.05$ (statistical significance).

to the infection. Seven of our patients were infected with COVID-19 after surgery, 3 of whom tested positive in the immediate postoperative period and 4 of whom contracted the disease after discharge. The 3 patients who contracted COVID-19 in the immediate postoperative period had undergone CABG. All of these patients developed a decrease in oxygen saturation with fever on day 2 or 3 . Repeat RTPCR revealed the diagnosis. All 3 underwent surgery at different time periods without overlap. These patients were moved to the COVID-19 ICU and were initially treated with high-flow oxygen and then with non-invasive ventilation using bilevel positive airway pressure. However, 1 of the patients gradually deteriorated and required invasive 
ventilation but did not improve and expired on postoperative day 7. For the other 2 patients, hypoxia gradually improved with medication and supportive oxygen therapy, and they were discharged to their homes. The remaining 4 patients developed COVID-19 infection after discharge, but they all recovered uneventfully and are doing well.

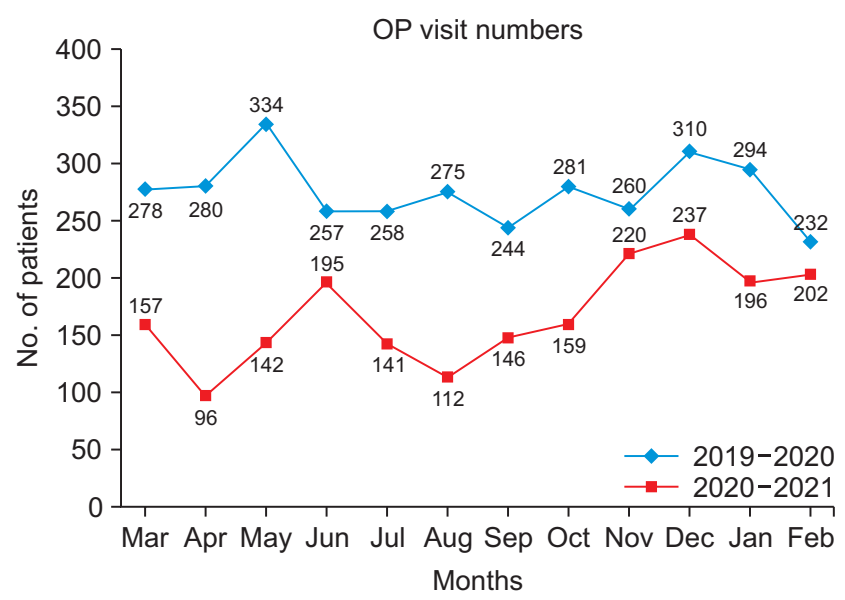

Fig. 2. Outpatient (OP) visit numbers in the pandemic (20202021) and pre-pandemic (2019-2020) periods.
Fourteen patients had been referred for surgery but were found to have COVID-19; hence, surgery was deferred. One of these patients died from sudden cardiac arrest while waiting for surgery for aortic stenosis. Two other patients recovered from COVID-19 but declined surgery. One patient underwent a percutaneous coronary intervention for coronary artery disease, so surgery was not performed. The remaining 10 patients underwent surgery at a median of 90 days after testing positive for COVID-19 (Table 5). Among the 10 patients with preoperative COVID-19 infection who underwent surgery after recovery from COVID, 1 middle-aged woman who underwent a valve-sparing aortic root replacement reported persistent dyspnea when walking in the postoperative ward. A computer tomogram of the pulmonary arteries showed evidence of segmental pulmonary embolism despite treatment with low molecular heparin, which we routinely administer to all patients postoperatively for deep vein thrombosis prophylaxis. She was treated medically with a therapeutic dose of anticoagulation and improved. One woman who underwent a descending aortic aneurysm repair had a prolonged postoperative recovery requiring ventilator support and tracheostomy and was eventually discharged to a local hospital for continued care. The other 8 had uneventful recovery periods.

Table 5. Outcomes of the 10 patients for whom surgery was delayed due to COVID-19

\begin{tabular}{|c|c|c|c|c|c|c|c|c|}
\hline $\begin{array}{l}\text { Patient } \\
\text { no. }\end{array}$ & $\begin{array}{l}\text { Age } \\
(y r)\end{array}$ & Sex & Diagnosis & Operation & $\begin{array}{l}\text { Time from COVID- } \\
19 \text { diagnosis to } \\
\text { surgery (day) }\end{array}$ & $\begin{array}{c}\text { Hospital } \\
\text { stay } \\
\text { (day) }\end{array}$ & Complications & Mortality \\
\hline 1 & 73 & M & Coronary artery disease & OPCAB & 46 & 10 & - & - \\
\hline 2 & 73 & M & $\begin{array}{l}\text { Degenerated bioprosthetic } \\
\text { aortic valve with aortic } \\
\text { regurgitation }\end{array}$ & Redo AVR & 31 & 11 & - & - \\
\hline 3 & 53 & $\mathrm{~F}$ & Aortic root aneurysm & $\begin{array}{l}\text { David } \\
\text { procedure }\end{array}$ & 57 & 24 & $\begin{array}{l}\text { Complete heart block: } \\
\text { PPI placed, pulmonary } \\
\text { embolism }\end{array}$ & - \\
\hline 4 & 69 & M & $\begin{array}{l}\text { Mitral valve prolapse, } \\
\text { mitral regurgitation }\end{array}$ & $\begin{array}{l}\text { Mitral valve } \\
\text { repair }\end{array}$ & 51 & 9 & - & - \\
\hline 5 & 71 & M & Coronary artery disease & OPCAB & 82 & 8 & - & - \\
\hline 6 & 50 & M & Coronary artery disease & OPCAB & 170 & 6 & - & - \\
\hline 7 & 75 & $\mathrm{~F}$ & DTA aneurysm & $\begin{array}{l}\text { DTA } \\
\text { aneurysm } \\
\text { repair }\end{array}$ & 100 & 45 & $\begin{array}{l}\text { Bloodstream infection, } \\
\text { prolonged ventilation- } \\
\text { tracheostomy }\end{array}$ & - \\
\hline 8 & 78 & M & Coronary artery disease & OPCAB & 99 & 14 & $\begin{array}{l}\text { Heart failure, R chest } \\
\text { tube insertion for } \\
\text { effusion }\end{array}$ & - \\
\hline 9 & 41 & M & $\begin{array}{l}\text { Infective endocarditis, } \\
\text { severe aortic regurgitation }\end{array}$ & AVR & 252 & 8 & - & - \\
\hline 10 & 57 & M & Coronary artery disease & OРCAB & 144 & 6 & - & - \\
\hline
\end{tabular}

COVID-19, coronavirus disease 2019; M, male; F, female; OPCAB, off-pump coronary artery bypass; AVR, aortic valve replacement; DTA, descending thoracic aorta; PPI, permanent pacemaker implantation. 


\section{Discussion}

Surgery and SARS-CoV-2 are interlinked. Surgery makes a patient susceptible to COVID-19 infection since, for one, it increases the risk of exposure to the infection in the hospital. Additionally, the chance of developing more severe complications of COVID-19 is relatively high due to the associated immunosuppressive response to surgery and the inflammatory cytokine storm associated with COVID-19 infection [12,13]. COVID-19-positive patients undergoing cardiac surgery are suspected to have very high mortality due to this inflammatory storm. Suspicion also exists that the presence of underlying cardiac disease can worsen COVID-19 infection, affecting the prognosis [14]. Hence, a general reluctance exists to perform cardiac surgery on COVID-19-positive patients. In an international multicenter cohort study of patients with perioperative COVID19 infection who underwent any procedure, the 30-day mortality rate was $23.8 \%$; among those who underwent cardiac surgery, that rate was $34 \%$ [15].

India is the country that has been the second most heavily affected by COVID-19, and Kerala, the state in which our center is located, is the second most affected state in the nation. During the first wave of COVID-19, although we had prepared a department protocol to defer elective cases to avoid burdening hospital resources in the case of an unprecedented COVID-19 surge to tier 3 numbers, the actual COVID-19 burden in the hospital did not reach alarming levels. We had decided to scale back our schedule early in the pandemic, but we also observed a decline in patient numbers, probably due to fear among patients to visit the hospital for evaluation. This led to a significant decrease in both surgical volume and outpatient follow-up visits. This experience resembles that reported in most centers around the world [16]. Notwithstanding, the continuing fear of the virus, the possibilities of asymptomatic patients or falsenegative test results, and the adverse effect of the virus on patients' cardiac status increased the fear that cardiac surgical outcomes would be affected [17]. However, the results of the present study show that despite the restrictions and concurrent delays in seeking care, as well as the new care pathways introduced with preoperative testing and quarantine before surgery, surgical outcomes did not change significantly during the pandemic period. In contrast, a report from another center comparing surgical outcomes for a shorter period at the beginning of the lockdown compared to a pre-lockdown period showed worse outcomes of surgery [18]. However, this study presents findings from a longer duration and hence is thought to represent a broad- er picture. A significant decrease in overall postoperative complications was observed during the pandemic period, perhaps due to more focused patient care associated with reduced patient volume. The decrease in surgical site infection may be attributable to restricted patient and bystander entry to the hospital during this period, as well as the higher rate of universal masking for all personnel involved in patient care [19]. We did not observe the anticipated increase in the incidence of patients with LV dysfunction presenting for $\mathrm{CABG}$ surgery compared to the previous year. This finding contrasts with the literature regarding an increase in the incidence of post-myocardial infarction complications during the COVID-19 period, and this may be due to the relatively small sample size or the patient population on which we focused [20]. These results should reassure both patients and, especially, the surgical community that with protocols in place, effective cardiac care can be delivered even in these trying times.

The outcomes of patients who had COVID-19 preoperatively, recovered, and were scheduled for cardiac surgery were good. This resembles the findings reported in the literature [21]. The postoperative detection of segmental pulmonary embolism in 1 woman was novel among these patients. The degree to which we can attribute this to a postCOVID-19 hypercoagulable status is uncertain, but it was the first time we had diagnosed a postoperative pulmonary embolism in a patient. The propensity for COVID-19 to cause thrombosis even in patients treated with thrombosis prophylaxis has been previously reported [22].

\section{Staff exposure to COVID-19}

Early in the pandemic period, a surgeon on our team contracted COVID-19, most likely after conducting an inhouse consultation for a CABG procedure after which the patient developed fever and diarrhea and was diagnosed with COVID-19. The rest of the surgeons, of whom the resident and physician assistant were first-degree contacts with the infected surgeon, had to quarantine as a result. Hence, the division was virtually shut down for a week, during which 1 of our pediatric cardiac surgical colleagues performed rounds on our postoperative patients. This experience caused us to stagger our work pattern and try to minimize close contact among surgeons for the next few months to prevent a similar closure of the division. Workflow disruptions due to staff shortages have also been seen elsewhere during the pandemic [8].

The known risks of waiting for surgery with a cardiac illness make it imperative to plan to continue cardiac sur- 
gery in these difficult times. The strong public healthcare system in Kerala, the proactive state government, and active community participation are probable reasons why our center, and the state as a whole, was able to endure the first COVID-19 wave without alarming results [23]. Provisional data from the United States show that heart disease remained the leading cause of death in 2020 and actually increased by $4.8 \%$ compared to the prior year, the highest increase in incidence since 2012 [24]. This highlights the critical nature of healthcare planning in restarting and conducting cardiac surgery and other such critical services so additional waves of the current pandemic and any future similar crises can be managed and collateral damage minimized as much as possible [25].

Health policy decision-makers and healthcare providers have struggled to determine the right balance between avoiding exposure to COVID-19 for patients with heart disease and other major comorbidities and the inherent risk of mortality and morbidity associated with not seeking appropriate and timely medical care out of fear of contracting COVID-19. It remains to be seen whether cardiac surgery practice will be impacted by the long-term effects of COVID-19, such as permanent lung damage due to fibrosis, possible cardiomyopathy related to myocarditis, and sequelae of thromboembolic disease [26]. The answers are not straightforward, and the ongoing pandemic will undoubtedly help frame future health policy decisions around similar major disease outbreaks. As such, we hope that these data will contribute to the framing of guidelines to deal with future crises.

\section{Conclusions}

The COVID-19 pandemic and associated social restrictions caused a substantial decrease in surgical volumes and outpatient visits. Despite this drop in numbers and occasional disruptions in service for COVID-19-positive patients, no significant change in patient outcomes was observed over this period. Heart disease remains the leading cause of mortality globally, and it is imperative that cardiac care continues during this and future global health crises. An acceptable cardiac standard of care can still be delivered during these difficult times.

\section{Conflict of interest}

No potential conflict of interest relevant to this article was reported.

\section{Acknowledgments}

We thank Ms. Renjitha Bhaskaran, MSc, Lecturer, Department of Biostatistics for help with the statistical analysis for this study.

\section{ORCID}

Kirun Gopal: https://orcid.org/0000-0002-9622-4230

Neethu Krishna: https://orcid.org/0000-0002-1834-8086

Rajesh Jose: https://orcid.org/0000-0002-2116-2821

Surya Sree Chitra Biju: https://orcid.org/0000-0001-9211-888X Jaya Suriya Pichandi: https://orcid.org/0000-0002-9693-0127

Praveen Kerala Varma: https://orcid.org/0000-0003-3978-2874

\section{References}

1. Wu J, Mamas MA, Mohamed MO, et al. Place and causes of acute cardiovascular mortality during the COVID-19 pandemic. Heart 2021;107:113-9.

2. Wadhera RK, Shen C, Gondi S, Chen S, Kazi DS, Yeh RW. Cardiovascular deaths during the COVID-19 pandemic in the United States. J Am Coll Cardiol 2021;77:159-69.

3. Zorzi A, Vio R, Rivezzi F, et al. Characteristics and hospital course of patients admitted for acute cardiovascular diseases during the coronavirus disease-19 outbreak. J Cardiovasc Med (Hagerstown) 2021;22:29-35.

4. De Rosa S, Spaccarotella C, Basso C, et al. Reduction of hospitalizations for myocardial infarction in Italy in the COVID-19 era. Eur Heart J 2020;41:2083-8.

5. Tam CF, Cheung KS, Lam S, et al. Impact of coronavirus disease 2019 (COVID-19) outbreak on ST-segment-elevation myocardial infarction care in Hong Kong, China. Circ Cardiovasc Qual Outcomes 2020;13:e006631.

6. Abdelaziz HK, Abdelrahman A, Nabi A, et al. Impact of COVID-19 pandemic on patients with ST-segment elevation myocardial infarction: insights from a British cardiac center. Am Heart J 2020;226:458 .

7. Wilson SJ, Connolly MJ, Elghamry Z, et al. Effect of the COVID-19 pandemic on ST-segment-elevation myocardial infarction presentations and in-hospital outcomes. Circ Cardiovasc Interv 2020;13: e009438

8. Osman F, Caplin N, Bashir M. COVID-19: the rising cost of cardiac surgery and disease. J Card Surg 2021;36:1593-6.

9. Vervoort D, Luc JG, Percy E, Hirji S, Lee R. Assessing the collateral damage of the novel coronavirus: a call to action for the postCOVID-19 era. Ann Thorac Surg 2020;110:757-60.

10. Hiremath CS, Yadava OP, Meharwal ZS, Iyer KS, Velayudhan B; COVID-19 Task Force of the Indian Association of Cardiovascu- 
lar-Thoracic Surgeons. IACTS guidelines: practice of cardiovascular and thoracic surgery in the COVID-19 era. Indian J Thorac Cardiovasc Surg 2020;36:1-13.

11. Haft JW, Atluri P, Ailawadi G, et al. Adult cardiac surgery during the COVID-19 pandemic: a tiered patient triage guidance statement. J Thorac Cardiovasc Surg 2020;160:452-5.

12. Besnier E, Tuech JJ, Schwarz L. We asked the experts: covid-19 outbreak: is there still a place for scheduled surgery?: "reflection from pathophysiological data". World J Surg 2020;44:1695-8.

13. Huang C, Wang Y, Li X, et al. Clinical features of patients infected with 2019 novel coronavirus in Wuhan, China. Lancet 2020;395: 497-506.

14. Li B, Yang J, Zhao F, et al. Prevalence and impact of cardiovascular metabolic diseases on COVID-19 in China. Clin Res Cardiol 2020; 109:531-8

15. COVIDSurg Collaborative. Mortality and pulmonary complications in patients undergoing surgery with perioperative $S A R S-C o V-2$ infection: an international cohort study. Lancet 2020;396:27-38.

16. Harky A, Chen R, Pullan M. Examining the impact of COVID-19 on cardiac surgery services: the lessons learned from this pandemic. J Card Surg 2020;35:2364-6.

17. Xiong TY, Redwood S, Prendergast B, Chen M. Coronaviruses and the cardiovascular system: acute and long-term implications. Eur Heart J 2020;41:1798-800.

18. Nader J, Anselmi A, Tomasi J, et al. Adult cardiac surgery during COVID-19 lockdown: impact on activity and outcomes in a high- volume centre. Arch Cardiovasc Dis 2021;114:364-70.

19. Losurdo P, Paiano L, Samardzic N, et al. Impact of lockdown for SARS-CoV-2 (COVID-19) on surgical site infection rates: a monocentric observational cohort study. Updates Surg 2020;72:1263-71.

20. Tomasoni D, Adamo M, Italia L, et al. Impact of COVID-2019 outbreak on prevalence, clinical presentation and outcomes of ST-elevation myocardial infarction. J Cardiovasc Med (Hagerstown) 2020;21: 874-81.

21. Sanders J, Akowuah E, Cooper J, et al. Cardiac surgery outcome during the COVID-19 pandemic: a retrospective review of the early experience in nine UK centres. J Cardiothorac Surg 2021;16:43.

22. Khan IH, Savarimuthu S, Leung MS, Harky A. The need to manage the risk of thromboembolism in COVID-19 patients. J Vasc Surg 2020;72:799-804.

23. Menon JC, Rakesh PS, John D, Thachathodiyl R, Banerjee A. What was right about Kerala's response to the COVID-19 pandemic? BMJ Glob Health 2020;5:e03212.

24. Ahmad FB, Anderson RN. The leading causes of death in the US for 2020. JAMA 2021;325:1829-30.

25. Chikwe J, Gaudino M, Hameed I, et al. Committee Recommendations for Resuming Cardiac Surgery Activity in the SARS-CoV-2 Era: guidance from an International Cardiac Surgery Consortium. Ann Thorac Surg 2020;110:725-32.

26. Young MN, Iribarne A, Malenka D. COVID-19 and cardiovascular health: this is a public service announcement. J Am Coll Cardiol 2021;77:170-2. 